\title{
Treatment of nasopharyngeal carcinoma by tomotherapy: five-year experience
}

Stephen Wan Leung ${ }^{1,2+}$ and Tsair-Fwu Lee 3*t $^{3 *}$

\begin{abstract}
Purpose: To analyze of survival curve and toxicity outcomes for patients treated for nasopharyngeal carcinoma (NPC) by intensity-modulated radiotherapy (IMRT) delivered by helical TomoTherapy (HT).

Materials and methods: Since May 2006, 72 patients with primary NPC were treated. In 67 cases PET-CT was used to help delineate the gross tumor volume (GTV); in 4 of these cases distant metastases in bone, mediastinal lymph nodes and unexpected small neck nodes were detected by high SUV uptake. 3, 22, 19, and 27 patients, respectively, had AJCC stage I to IV disease. Patients received a median total dose of 72 Gy to the GTV, 64.8 Gy to the elective PTV, and 54 Gy to the clinically negative neck region.

Results: At a median follow-up of 41 months (range 0.2 to 67 months), no patient has recurred locally. Two patients with stage $\mathrm{ll} b$ disease, both of whom received chemotherapy, recurred regionally. Ten patients developed distant metastases. One died from progressive disease with initial proved bony metastasis. Two patients with stage Illb disease, both of whom received chemotherapy, experienced neck node recurrence. 5-year locoregional control rate was 97\%; freedom from distant metastases was $84.6 \%$ at 5 years. No evidence of disease was detected in 13 early stage $(\mathrm{l} / \mathrm{ll} \mathrm{a} / \mathrm{ll} \mathrm{b})$ patients who did not receive chemotherapy. Acute grade 3 toxicity occurred in four patients and grade 4 in two patients. Late toxicities were low, with no grade $3+$ xerostomia, grade 2 xerostomia in two patients (3\%), and grade 3 hearing loss in two patients (3\%).

Conclusions: HT resulted in excellent long-term disease control and survival in heterogeneous NPC patients. Generally mild acute and late toxicity, with low rates of xerostomia, were obtained. Image-guided HT offers the ability to deliver conformal, OAR-sparing dose distributions to a wide variety of NPC patients with good long-term clinical outcomes.
\end{abstract}

\section{Introduction}

In 2002 the United States Food and Drug Administration (FDA) approved an innovative radiation therapy device called Helical TomoTherapy (HT; Accuray Incorporated, Sunnyvale, CA). The 3-dimensional imaging provided by megavoltage CT allows for accurate patient setup and precise radiation delivery [1-3]. The helical delivery of intensity-modulated radiotherapy (IMRT) allows excellent conformality and homogeneity of the radiation dose distribution [4-9]. The advantage of its unique approach to radiation delivery is especially clear for complicated dose

\footnotetext{
* Correspondence: tflee@kuas.edu.tw

${ }^{\dagger}$ Equal contributors

${ }^{3}$ Department of Electronics Engineering, Medical Physics and Informatics Library, National Kaohsiung University of Applied Sciences, Kaohsiung, Taiwan

Full list of author information is available at the end of the article
}

distributions involving multiple planning target volumes (PTVs) and organs at risk (OAR), such as those often required for adequate and safe treatment of head and neck cancer $[5,9]$.

Considerable research with the HT system has focused on dosimetry of head and neck plans relative to other radiation therapy platforms. In general these studies indicate that HT plans are superior to step-and-shoot (SaS) IMRT plans in terms of target coverage and OAR sparing, or superior to or comparable to arcing IMRT plans, depending on the parameter under consideration. For example, Lee et al. [9] showed that HT plans were more conformal and homogeneous than SaS-IMRT plans, and that the dose to most of the OARs was lower in HT plans (in patients without skull base infiltration HT did not lower the dose to the optic chiasm). Similar outcomes have been obtained in several other studies

\section{Biomed Central}


[6,8,10-12]. Arcing IMRT plans usually fared better than non-arcing IMRT when compared with HT plans; Wiezorek et al. obtained generally superior OAR sparing with arcing IMRT plans in their study of 10 head and neck cancer patients, but concluded the overall plan quality was best for HT.

The first clinical outcomes for HT treatment of head and neck cancer have recently been published. Kodaira et al. [13] used HT to treat 20 pts with NPC, some node-positive and with metastases, most of whom underwent chemotherapy. The progression-free and overall survival rates at 10 months were $79.7 \%$ and 95\%, respectively. Parotid function assessed by quantitative salivary scintigraphy showed a drop in maximal excretion ratio at 3 months which returned to near baseline by one year; at 3 months $61.1 \%$ of patients had Grade 2 and $38.9 \%$ had Grade 1 xerostomia, but by 9 months, Grade 2 xerostomia was observed in $26.7 \%$ of patients, Grade 1 in 66.7\%, and Grade 0 in the rest. Most recently Chen et al. [14] studied patients treated for nasopharyngeal cancer (NPC) using HT or IMRT delivered via segmental multileaf collimator. They replicated the dosimetric superiority of HT, showing significant reductions in dose to parotids, temporal lobes, and ipsilateral ear structures. Overall survival and disease control for the two groups was nearly identical. Although RTOGgraded scoring of late xerostomia was not significantly different ( $13 \%$ for IMRT vs $7 \%$ for HT), $38 \%$ of IMRT patients (7 of 16 patients) reported "too little" or "no" saliva using the University of Washington quality of life survey (UW-QOL), while 7\% (1 of 14 patients) of HT patients reported too little saliva. The authors concluded that the dosimetric differences between HT and SaSIMRT did, indeed, result in improved clinical outcomes. Despite these instances, published clinical data on HT for NPC is sparse. Here we present long-term (5 year) clinical outcomes of HT for 72 patients with nasopharyngeal cancer.

\section{Materials and methods \\ Patients}

Since May 2006, 72 patients (59 men and 13 women) with primary NPC were treated with curative RT by HT in the Department of Radiation Oncology, Kaohsiung Yuan's General Hospital. Patient characteristics are indicated in Table 1. The median age was 46.5 years (range 23 to 80 years). The distribution of clinical stages according to the American Joint of Cancer Committee (AJCC) staging system published in 1997 was 3 patients $(4 \%)$ at Stage I, $23(32 \%)$ at Stage II, $19(26 \%)$ at Stage III, and 27 patients (37.5\%) at Stage IV. Forty-five patients (62.5\%) received concomitant chemotherapy, and 27 patients $(37.5 \%)$ did not. This study was approved
Table 1 Patient characteristics $(n=72)$

\begin{tabular}{|c|c|}
\hline Variables & Number (\%) \\
\hline Age, median years (range) & $46.5(23-80)$ \\
\hline \multicolumn{2}{|l|}{ Gender } \\
\hline Male & 59 (82\%) \\
\hline Female & $13(18 \%)$ \\
\hline \multicolumn{2}{|l|}{ Smoker } \\
\hline Yes & $30(41.5 \%)$ \\
\hline No & $40(55.5 \%)$ \\
\hline Unknown & $2(3 \%)$ \\
\hline \multicolumn{2}{|l|}{ AJCC stage } \\
\hline । & $3(4 \%)$ \\
\hline$\| \mathrm{a} / \mathrm{b}$ & $7 / 16$ (total 32\%) \\
\hline III & $19(26 \%)$ \\
\hline IV a/b/c & 6/14/7 (total 37.5\%) \\
\hline \multicolumn{2}{|l|}{ T stage } \\
\hline $\mathrm{T} 1$ & $30(41.5 \%)$ \\
\hline $\mathrm{T} 2$ & $11(15 \%)$ \\
\hline T2a & $2(3 \%)$ \\
\hline $\mathrm{T} 2 \mathrm{~b}$ & $6(8 \%)$ \\
\hline T3 & $15(21 \%)$ \\
\hline T4 & $8(11 \%)$ \\
\hline \multicolumn{2}{|l|}{$\mathrm{N}$ stage } \\
\hline No & $13(18 \%)$ \\
\hline N1 & $24(33 \%)$ \\
\hline N2 & $20(28 \%)$ \\
\hline N3 & 15 (21\%) \\
\hline \multicolumn{2}{|c|}{ Combination with chemotherapy } \\
\hline Yes & $45(62.5 \%)$ \\
\hline No & $27(37.5 \%)$ \\
\hline \multicolumn{2}{|l|}{ Brachytherapy } \\
\hline \multirow[t]{5}{*}{ Yes } & 15 (21\%) \\
\hline & Stage I: 1 patients \\
\hline & Stage lla 3 patients \\
\hline & Stage IIb 7 patients \\
\hline & Stage III 4 patients \\
\hline No & 57 (79\%) \\
\hline \multicolumn{2}{|l|}{ PET/CT fusion } \\
\hline Yes & 67 (93\%) \\
\hline No & $5(7 \%)$ \\
\hline
\end{tabular}

AJCC, American Joint of Cancer Committee in 2007.

by the Medical Ethics Committee of the Kaohsiung Yuan's General Hospital (YUAN-IRB20120328B). Written informed consent was obtained from the patient for publication of this report and any accompanying images. 


\section{Treatment planning}

All patients were immobilized in a tailor-made thermoplastic cast from head to shoulders, and $3 \mathrm{~mm}$-thickness CT (12 patients) and/or PET/CT (67 patients; Siemens Biograph LSO PET/CT) scan slices of the head and neck were obtained to be used for localization of targets and organs at risk (OARs). CT or PET/CT image sets were then transferred to and fused in the Pinnacle ${ }^{3}$ planning system (Phillips Healthcare, The Netherlands) for contouring. Contours were transferred to HT for IMRT inverse planning. In four of the patients simulated with $\mathrm{PET} / \mathrm{CT}$, distant metastases in bone, mediastinal lymph nodes and unexpected small neck nodes were detected by high SUV uptake.

The gross tumour volume (GTV), including the macroscopic primary cancer and nodes greater than $1 \mathrm{~cm}$ in diameter or nodes with necrotic centres, was used for all plans. RT was delivered using a sequential IMRT technique and three cone-down target volumes were defined for each patient. According to the RTOG 0225 and previous studies [15-18], for a typical case by using the prescribed dose equals to $72 \mathrm{~Gy}$, the clinical target volume (CTV) for the delivery of absorbed dose of 72 Gy $\left(\mathrm{CTV}_{72}=\mathrm{GTV}+5 \mathrm{~mm}\right.$ margin $)$ is defined using an isotropic margin of $5 \mathrm{~mm}$ around the GTV. The clinical target volume for 64.8 Gy delivery $\left(\mathrm{CTV}_{64.8}\right)$ equals to the $\mathrm{CTV}_{72}+5 \mathrm{~mm}$ margin plus areas at risk for microscopic involvement, including the entire nasopharynx, retropharyngeal nodal regions, skull base, clivus, pterygoid fossae, parapharyngeal space, sphenoid sinus (in T3-T4 disease, the entire sphenoid sinus), the posterior third of the nasal cavity/maxillary sinuses that includes the pterygopalatine fossae, and levels I through $\mathrm{V}$ nodal regions. The cavernous sinus should be included in high-risk patients (T3, T4, bulky disease involving the roof of the nasopharynx). The clinical target volume for 54 Gy $\left(\mathrm{CTV}_{54}\right)$ includes the clinically negative low neck regions. Safety margins between the CTV and planning target volume (PTV) of $5 \mathrm{~mm}$ were used for $\mathrm{CTV}_{72}$ and $\mathrm{CTV}_{64.8}$ to account for patient setup error and motion uncertainties, but in areas in which the GTV or the CTV was adjacent to critical normal structures (i.e., the brainstem) the margin was reduced to $1 \mathrm{~mm}$; no safety margin was used for the generation of planning target volume$54 \mathrm{~Gy}\left(\mathrm{PTV}_{54}\right)$. In our study cohort, the median (and modal) prescribed dose was 72 Gy to the PTV (39.6 Gy to 75.6 Gy) (PTV 72$), 64.8$ Gy to the elective PTV(PTV P4.8 $\left._{5}\right)$, and 54 Gy to the clinically negative neck region $\left(\mathrm{PTV}_{54}\right)$ with a daily fraction size of 1.8 Gy in terms of three conedown treatment schemes. Typically for the prescribed dose equal to $72 \mathrm{~Gy}$, the prescription dose was set to (a) 28 fractions containing all three PTVs, (b) eight fractions containing $\mathrm{PTV}_{72}$ and $\mathrm{PTV}_{64.8}$, and (c) four fractions treated with $\mathrm{PTV}_{72}$ alone. OARs included seven serial- type organs (serial OARs; brainstem, spinal cord, lenses, eyes, optic nerves, chiasm, and mandible) and three parallel-type organs (parallel OARs; parotids, submandibular glands, and oral cavity). Maximum doses to OARs were optimized on an individual basis without compromising the PTV coverage, with at least $95 \%$ of the PTV receiving the minimum prescribed dose. Fifteen patients (21\%) also received intracavitary brachytherapy delivered to the GTV, 3-6 Gy in 1-2 fractions delivered twice per week, the dose prescribed to $2 \mathrm{~cm}$ off the source axis.

\section{Treatment delivery}

Treatment was delivered in five fractions per week. Daily patient setup began with laser-based positioning followed by MVCT scanning using the coarse setting. Offsets were used to correct patient positioning. IMRT was delivered with a $2.5 \mathrm{~cm}$ field width, a pitch of 0.3 , and a modulation factor of 2.5. These settings have been shown in prior work to produce plans that are superior to 7-field step-and-shoot IMRT plans [9]. Repeat CT simulation occurred 2 to 3 times during treatment to allow cone-down boosting and plan adaptation to meet dosimetric goals for PTVs and maintain dose constraints to OARs.

Concurrent chemotherapy was administered in 45 (62.5\%) patients. For most patients this consisted of cisplatin and fluorouracil; some patients were treated with combinations that included oxaliplatin, tegafur-uracil (Ufur), hydroxyurea, carboplatin, or erbitux.

\section{Patients follow-up}

Patients were evaluated for disease control, survival, and toxicity according to RTOC toxicity criteria at 2-month intervals for the first 2 years, at 3- to 6-month intervals between the third and fifth years, and at 1-year intervals thereafter. Follow-up examination of the primary tumor was assessed by fiberoptic endoscopy at every visit. CT scan, bone scans, chest radiography, and liver sonography were performed every 6 months in the first 2 years and annually thereafter.

\section{Results}

\section{Dosimetry}

Highly conformal, homogeneous treatment plans were generated for HT [9]. Table 2 shows that dose constraints for organs at risk were generally maintained. In particular, both ipsilateral and contralateral parotid glands were held below the 26 Gy dose constraints. Doses to OARS were always less than doses recommended under RTOG protocol 0615.

\section{Disease control}

At a median follow-up of 41 months, no patient has recurred locally. Two patients with stage IIb disease, 
Table 2 The mean or maximum doses to organs at risk (OARs)

\begin{tabular}{lll}
\hline OARs & Mean value \pm SD (Gy) & Range (Gy) \\
\hline Serial & Maximum dose & \\
Brain stem & $50.53 \pm 1.78$ & $46.40-52.75$ \\
Spinal cord & $39.95 \pm 1.95$ & $35.86-42.90$ \\
Ipsi-lateral lens & $3.78 \pm 0.78$ & $2.63-4.70$ \\
Contra-lateral lens & $3.58 \pm 0.70$ & $2.60-4.60$ \\
Ipsi-lateral eye & $11.19 \pm 3.10$ & $6.76-17.69$ \\
Contra-lateral eye & $10.38 \pm 2.51$ & $6.53-15.86$ \\
Mandible & $63.64 \pm 1.72$ & $60.17-65.44$ \\
Chiasm & $38.20 \pm 4.67$ & $15.20-46.50$ \\
Parallel & Mean dose & \\
Ipsi-lateral parotid gland & $22.07 \pm 1.66$ & $19.18-24.99$ \\
Contra-lateral parotid gland & $20.46 \pm 2.27$ & $15.48-24.56$ \\
Submandibular glands & $31.92 \pm 2.65$ & $27.85-37.09$ \\
Oral cavity & $32.49 \pm 6.09$ & $22.19-41.06$ \\
\hline
\end{tabular}

both of whom received chemotherapy, experienced neck node recurrence. Kaplan-Meier (KM) analysis resulted in a 5-year estimate of locoregional control of 97\% (see Figure 1). One of these patients was treated surgically and is currently without evidence of disease; the other was lost to follow-up. Ten patients had been proved for distant metastasis; KM freedom from distant metastases was $84.6 \%$ at 5 years (Figure 1). Metastases occurred to bone, lung, and liver, and 5 patients presented with multiple metastatic sites. One patient died for disease

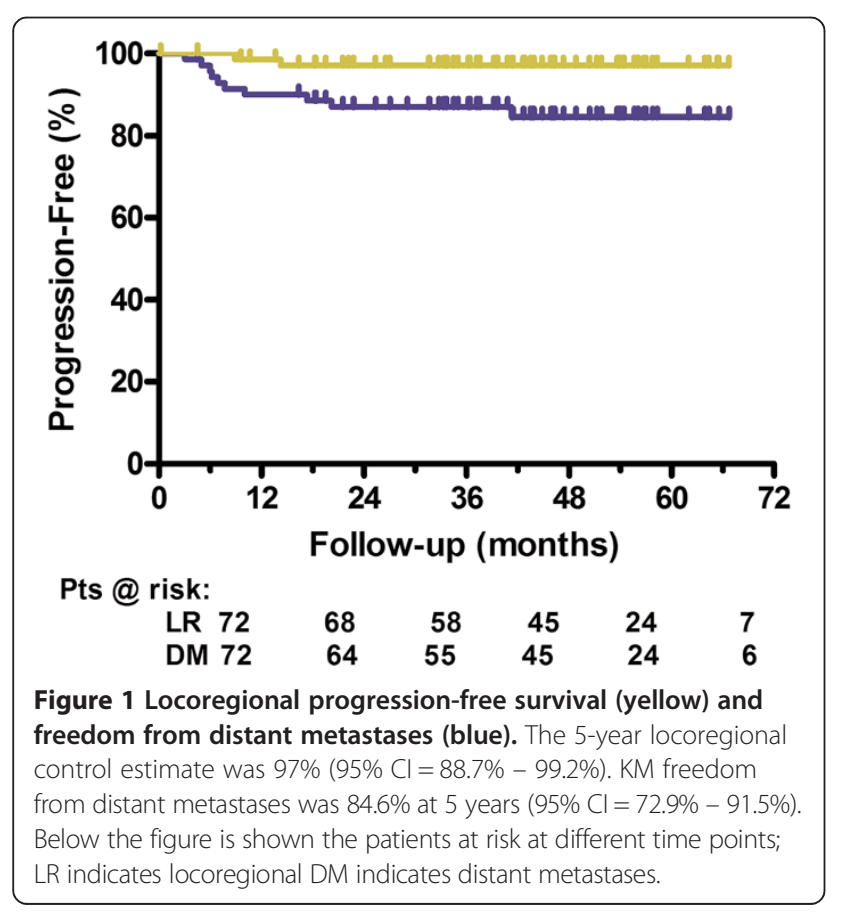

out of control with initial proved bony metastasis. No evidence of disease was detected in 13 early stage (I/IIa/ IIb) patients who did not receive chemotherapy. Seven patients have died of cancer; overall survival at 40 months mean follow-up was $90 \%$.

\section{Toxicity}

Most patients experienced no serious acute toxicity (Table 3). RTOG Grade 2 acute mucositis was observed in 6 patients (8\%), Grade 3 in 3 patients (4\%), and Grade 4 in 1 patient (1\%). Grade 2 dermatitis occurred in 8 patients (11\%), Grade 3 in no patients, and Grade 4 in 1 patient (1\%). All patients with Grade 3 and 4 acute toxicities were also treated concurrently with chemotherapy. Late toxicity was generally very mild, with Grade 2 xerostomia in 2 patients (3\%) and no Grade 3+ xerostomia. Grade 2 dysphagia occurred in 1 patient, with no Grade 3+ dysphagia. Grade 3 hearing loss was observed in 2 patients (3\%) who also received concurrent chemotherapy.

\section{Discussion}

The dosimetric advantages of HT for head and neck cancer have been reported in numerous publications, but few studies report on clinical outcomes of HT. The current study is among the largest, with the longest follow-up, reported in the literature to date. Its results demonstrate outstanding long-term disease control with acceptable levels of toxicity for NPC treated by NPC.

By allowing safe dose escalation to high-risk regions and simultaneous broad-field treatment of low-risk regions, IMRT has, arguably, enhanced NPC treatment efficacy and safety [17]. Lee et al. reported results from a multi-institutional trial of 68 patients with NPC treated with IMRT with or without chemotherapy [19]. Most patients received 70 Gy to the GTV and 59.4 Gy to subclinical regions. At a median follow-up of 2.6 years the 2-year estimate of local control was $92.6 \%$, progressionfree survival was $72.7 \%$, and overall survival $80.2 \%$. Grade 4 acute toxicities were observed in $11.8 \%$ and

Table 3 Acute and late toxicity (RTOG grading criteria)

\begin{tabular}{lccccc}
\hline & \multicolumn{5}{c}{ Grade } \\
\cline { 2 - 6 } Acute toxicity (N/\%) & $\mathbf{0}$ & $\mathbf{1}$ & $\mathbf{2}$ & $\mathbf{3}$ & $\mathbf{4}$ \\
\hline Mucositis & $42(58)$ & $20(28)$ & $6(8)$ & $3(4)^{*}$ & $1(1)^{*}$ \\
Dermatitis & $13(18)$ & $50(69)$ & $8(11)$ & $0(0)$ & $1(1)^{* *}$ \\
Late Toxicity (N/\%) & 0 & 1 & 2 & 3 & 4 \\
Xerostomia & $21(29)$ & $49(68)$ & $2(3)$ & $0(0)$ & $0(0)$ \\
Dysphagia & $65(90)$ & $6(8)$ & $1(1)$ & $0(0)$ & $0(0)$ \\
Hearing loss & $35(49)$ & $34(47)$ & $1(1)$ & $2(3)$ & $0(0)$ \\
Other & $72(100)$ & $0(0)$ & $0(0)$ & $0(0)$ & $0(0)$ \\
\hline
\end{tabular}

*Patients received cisplatin, fluorouracil, Ufur.

**Patient received oxaliplatin. 
Grade 3 in $61.8 \%$ of patients. Grade 2 xerostomia at 1 year was present in $13.5 \%$. Wolden et al. [20] treated 74 patients to 70 Gy with GTV boosting; most patients received concurrent and adjuvant platinum-based chemotherapy. At a median follow-up of 35 months the 3-year actuarial local control estimate was 91\%; progression-free survival and overall survival was $67 \%$ and $83 \%$, respectively. Long-term Grade 2 xerostomia occurred in $32 \%$ of patients (no Grade 3). Similar rates of long-term local disease control and overall survival, with Grade 2-3 xerostomia related to parotid dose [21], were reported by others using IMRT [21,22].

More recently reports of HT for locally advanced NPC have begun to appear. Kodaira et al. [13] treated 20 patients to 70 Gy to the tumor PTV and 54 Gy to the nodal PTV in 35 fractions. At a median follow-up of 10.3 months, 2 patients developed recurrent disease, in the liver in one patient and in a lymph node included in the tumor PTV for another. Progression-free and overall survival was $79.7 \%$ and $95 \%$, respectively. Shueng et al. [23] followed 28 patients to a median of 33 months after neoadjuvant chemotherapy followed by concurrent chemoradiation including HT to 70 Gy to the tumor and positive nodes. The 3-year local, regional, locoregional control and distant metastasis rate were $92.4 \%$, $95.7 \%, 88.4 \%$, and $78.0 \%$, respectively, and overall survival was $83.5 \%$. Toxicities generally occurred at a low rate, and late Grade 2 xerostomia was observed in $14 \%$ of patients (no Grade 3+). Ren et al. [24] treated 73 patients, 24 with radiation only, with $70-74$ Gy in 33 fractions to tumor PTV (primary tumor and positive lymph nodes), with 60-62.7 Gy a high-risk and 52-56 Gy to a low-risk PTV. At a median follow-up of 14.8 months the 1-year relapse-free survival, distant metastasis-free survival and overall survival were $95.6 \%, 97.2 \%$ and $94.8 \%$, respectively. By one year post-radiotherapy no patient reported Grade $2+$ xerostomia. In this context the current results compare favorably; at a median of 41 months follow-up, with several patients followed beyond 4 and 5 years, no patient recurred locally. Two regional recurrences and 10 distant metastases were obtained, and to date seven deaths due to cancer has occurred. Mean parotid doses were very low22.07 Gy and 20.46 Gy to the ipsilateral and contralateral glands, respectively-and late Grade 2+ xerostomia occurred in only $3 \%$ of patients. We conclude that the ability of HT to effectively target multiple regions of differing disease burden within the head and neck field while maximally sparing OARs, combined with daily MVCTguided patient setup accuracy and a rational regimen of systemic treatment, results in robust disease control with limited toxicity.

Although local control and survival have been enhanced by the introduction of IMRT into NPC treatment, the pattern of recurrence continues to show a relatively high rate of distant metastases $[17,20,21,23]$, suggesting the continuing need for more effective systemic therapies. In addition, it cannot be determined whether the generally superior dose-sculpting capability of HT relative to more conventional IMRT leads to improved disease control and toxicity outcomes. A recent paper by Chen et al. [14] reported on a comparison of 16 patients treated with IMRT delivered by sequential multileaf collimator and 14 with HT. Survival and disease control were identical between groups; 2-year local control was $87 \%$ in both groups, and overall survival did not differ significantly. The rate of xerostomia at any point in the late setting did not differ significantly between groups; at last follow-up, however, $38 \%$ of IMRT patients reported "too little" or "no" saliva, versus $7 \%$ of HT patients. Thus, recovery from xerostomia occurred more readily in the HT group. In a study by our group of quality-of-life between IMRT and HT patients, substantial improvements in saliva and oropharyngeal QOL variables were obtained with HT (in preparation). Thus, although for many patients superior HT treatment plans may have little effect on outcomes, for a significant subset the ability to easily reduce dose to parotids and other structures can be important for longterm QOL.

Potential limitation for this study may exist. Xerostomia related symptoms were usually cited as the most prevalent complications in NPC survivors post radiotherapy and patient-reported xerostomia has been found to significantly correlate with mean dose to the parotid glands and the minor salivary glands. Assessment of treatment-related toxicity by the well-validated healthrelated quality of life questionnaire rather than RTOC toxicity criteria would provide more informative data for the comparison among different studies.

The outcomes of the present study require additional long-term follow-up to assess the robustness of the promising disease control and low toxicity observed to date. In addition, the single-institution nature of the current study suggests a need to expand the HT methodology employed here to different patient populations in different institutions. In the meantime we are encouraged by the relatively low toxicity and excellent disease control with HT and, in selected patients, chemotherapy, to carry on with this approach to treating patients with locally advanced nasopharyngeal carcinoma.

\section{Conclusions}

Modern, advanced technology has brought the radiation oncology of head and neck cancer to a new era. We can generate better conformity and homogeneity than ever. In our clinical practice, acute and late complications after HT for NPC are definitely fewer and milder compared with our conventional IMRT technique. Helical TomoTherapy appears to have the advantages compared 
to IMRT for NPC treatment plans in terms of dosimetric distribution, and quality of life, local control and survival for our patients. Nevertheless, HT appears to have dosimetric advantages compared to IMRT which might translate into a significant benefit for the patients in clinical practice. To our knowledge, charged particles, such as proton beam or carbon ion beam, may have the best dosimetry for NPC plans. Comparison of the treatment outcomes of TomoTherapy to that of charged particles would be an interesting issue and warrants further investigation.

\section{Competing interests}

The authors declare that they have no competing interests.

\section{Authors' contributions}

SWL: original idea, study design, data collection and writing of manuscript. TFL: study design, statistical analysis, technical supports and correspondence. Both authors read and approved the final manuscript.

\section{Acknowledgments}

The authors thank David W Schaal, PhD. for editorial assistance. This study was supported financially, in part, by grant from the National Science Council (NSC), Taiwan, ROC. (NSC-101-2221-E-151-007-MY3). The authors thank the reviewers for their helpful comments on the original manuscript.

\section{Author details}

'Department of Radiation Oncology, Kaohsiung Yuan's General Hospital, Kaohsiung, Taiwan. ${ }^{2}$ Department of Radiological Technology, Central Taiwan University of Science and Technology, Taichuug, Taiwan. ${ }^{3}$ Department of Electronics Engineering, Medical Physics and Informatics Library, National Kaohsiung University of Applied Sciences, Kaohsiung, Taiwan.

Received: 22 February 2013 Accepted: 23 April 2013

Published: 1 May 2013

\section{References}

1. Langen KM, Zhang Y, Andrews RD, Hurley ME, Meeks SL, Poole DO, Willoughby TR, Kupelian PA: Initial experience with megavoltage (MV) CT guidance for daily prostate alignments. Int I Radiat Oncol Biol Phys 2005, 62(5):1517-1524.

2. Meeks SL, Harmon JF Jr, Langen KM, Willoughby TR, Wagner TH, Kupelian PA: Performance characterization of megavoltage computed tomography imaging on a helical tomotherapy unit. Med Phys 2005, 32(8):2673-2681.

3. Zeidan OA, Langen KM, Meeks SL, Manon RR, Wagner TH, Willoughby TR, Jenkins DW, Kupelian PA: Evaluation of image-guidance protocols in the treatment of head and neck cancers. Int I Radiat Oncol Biol Phys 2007, 67(3):670-677.

4. Fiorino C, Dell'Oca I, Pierelli A, Broggi S, De Martin E, Di Muzio N, Longobardi B, Fazio F, Calandrino R: Significant improvement in normal tissue sparing and target coverage for head and neck cancer by means of helical tomotherapy. Radiother Oncol 2006, 78(3):276-282.

5. Cao D, Holmes TW, Afghan MK, Shepard DM: Comparison of plan quality provided by intensity-modulated arc therapy and helical tomotherapy. Int J Radiat Oncol Biol Phys 2007, 69(1):240-250.

6. Murthy V, Master Z, Gupta T, Ghosh-Laskar S, Budrukkar A, Phurailatpam R, Agarwal J: Helical tomotherapy for head and neck squamous cell carcinoma: dosimetric comparison with linear accelerator-based stepand-shoot IMRT. J Cancer Res Ther 2010, 6(2):194-198.

7. Rong Y, Tang G, Welsh JS, Mohiuddin MM, Paliwal B, Yu CX: Helical tomotherapy versus single-arc intensity-modulated arc therapy: a collaborative dosimetric comparison between two institutions. Int J Radiat Oncol Biol Phys 2011, 81(1):284-296.

8. Wiezorek T, Brachwitz T, Georg D, Blank E, Fotina I, Habl G, Kretschmer M, Lutters G, Salz H, Schubert K, et al: Rotational IMRT techniques compared to fixed gantry IMRT and tomotherapy: multi-institutional planning study for head-and-neck cases. Radiat Oncol 2011, 6:20.
9. Lee TF, Fang FM, Chao PJ, Su TJ, Wang LK, Leung SW: Dosimetric comparisons of helical tomotherapy and step-and-shoot intensitymodulated radiotherapy in nasopharyngeal carcinoma. Radiother Oncol 2008, 89(1):89-96.

10. Mavroidis P, Ferreira BC, Shi C, Lind BK, Papanikolaou N: Treatment plan comparison between helical tomotherapy and MLC-based IMRT using radiobiological measures. Phys Med Biol 2007, 52(13):3817-3836.

11. Shi C, Penagaricano J, Papanikolaou N: Comparison of IMRT treatment plans between linac and helical tomotherapy based on integral dose and inhomogeneity index. Med Dosim 2008, 33(3):215-221.

12. van Vulpen $\mathrm{M}$, Field $\mathrm{C}$, Raaijmakers $\mathrm{CP}$, Parliament MB, Terhaard $\mathrm{CH}$, MacKenzie MA, Scrimger R, Lagendijk JJ, Fallone BG: Comparing step-and-shoot IMRT with dynamic helical tomotherapy IMRT plans for head-and-neck cancer. Int J Radiat Oncol Biol Phys 2005, 62(5):1535-1539.

13. Kodaira T, Tomita N, Tachibana H, Nakamura T, Nakahara R, Inokuchi H, Fuwa N: Aichi cancer center initial experience of intensity modulated radiation therapy for nasopharyngeal cancer using helical tomotherapy. Int J Radiat Oncol Biol Phys 2009, 73(4):1129-1134.

14. Chen AM, Yang CC, Marsano J, Liu T, Purdy JA: Intensity-modulated radiotherapy for nasopharyngeal carcinoma: improvement of the therapeutic ratio with helical tomotherapy versus segmental multileaf collimator-based techniques. Br J Radiol 2012, 85(1016):e537-e543.

15. Lee T-F, Chao P-J, Ting H-M, Lo S-H, Wang Y-W, Tuan C-C, Fang F-M, Su T-J: Comparative analysis of SmartArc-based dual arc volumetric-modulated arc radiotherapy (VMAT) versus intensity-modulated radiotherapy (IMRT) for nasopharyngeal carcinoma. J Appl Clin Med Phys 2011, 12(4):158-174.

16. Lee T-F, Ting H-M, Chao P-J, Fang F-M: Dual Arc volumetric-modulated Arc radiotherapy (VMAT) of nasopharyngeal carcinomas: a simultaneous integrated boost treatment plan comparison with intensity-modulated radiotherapies and single arc VMAT. Clin Oncol 2012, 24(3):196-207.

17. Lee N, Harris J, Garden AS, Straube W, Glisson B, Xia P, Bosch W, Morrison WH, Quivey J, Thorstad W, et al: Intensity-modulated radiation therapy with or without chemotherapy for nasopharyngeal carcinoma: radiation therapy oncology group phase II trial 0225. J Clin Oncol 2009, 27(22):3684-3690.

18. Kong L, Lu JJ, Lee N: Advances in the technology of radiation therapy for nasopharyngeal carcinoma. In Nasopharyngeal Cancer. 16th edition. Springer; 2010:197-211

19. Lee C, Langen KM, Lu W, Haimerl J, Schnarr E, Ruchala KJ, Olivera GH, Meeks SL, Kupelian PA, Shellenberger TD, et al: Assessment of parotid gland dose changes during head and neck cancer radiotherapy using daily megavoltage computed tomography and deformable image registration. Int J Radiat Oncol Biol Phys 2008, 71(5):1563-1571.

20. Wolden SL, Chen WC, Pfister DG, Kraus DH, Berry SL, Zelefsky MJ: Intensitymodulated radiation therapy (IMRT) for nasopharynx cancer: update of the Memorial Sloan-Kettering experience. Int I Radiat Oncol Biol Phys 2006, 64(1):57-62.

21. Kam MK, Teo PM, Chau RM, Cheung KY, Choi PH, Kwan WH, Leung SF, Zee B, Chan AT: Treatment of nasopharyngeal carcinoma with intensitymodulated radiotherapy: the Hong Kong experience. Int J Radiat Oncol Biol Phys 2004, 60(5):1440-1450.

22. Kwong DL, Pow EH, Sham JS, McMillan AS, Leung LH, Leung WK, Chua DT, Cheng AC, Wu PM, Au GK: Intensity-modulated radiotherapy for earlystage nasopharyngeal carcinoma: a prospective study on disease control and preservation of salivary function. Cancer 2004, 101(7):1584-1593.

23. Shueng PW, Shen BJ, Wu LJ, Liao L, Hsiao CH, Lin YC, Cheng PW, Lo WC, Jen YM, Hsieh $\mathrm{CH}$ : Concurrent image-guided intensity modulated radiotherapy and chemotherapy following neoadjuvant chemotherapy for locally advanced nasopharyngeal carcinoma. Radiat Oncol 2011, 6:95.

24. Ren G, Du L, Ma L, Feng LC, Zhou GX, Qu BL, Xu SP, Xie CB, Ou GM, Li F, et al: Clinical observation of 73 nasopharyngeal carcinoma patients treated by helical tomotherapy: the China experience. Technol Cancer Res Treat 2011, 10(3):259-266.

\section{doi:10.1186/1748-717X-8-107}

Cite this article as: Leung and Lee: Treatment of nasopharyngeal carcinoma by tomotherapy: five-year experience. Radiation Oncology 2013 8:107. 